\title{
NUMERICAL SIMULATION OF WAVINESS IN A CIRCULAR EHL CONTACT, UNDER ROLLING/SLIDING
}

\author{
(. H. Venner ${ }^{a b}$ and A.A. Lubrecht ${ }^{c}$ \\ ${ }^{a}$ University of Twente, fac. of Mechanical Engineering, Enschede, The Netherlands. \\ ${ }^{b}$ Dep. of Appl. Math., Weizmann Institute of Science, Rehovot, Israel. \\ ${ }^{\complement}$ Laboratoire de Mécanique des Contacts, URA CNRS 856, INSA de Lyon, France.
}

Surface roughness and/or surface imperfections are well known to significantly affect the performance of concentrated contacts. Helped by improvements in computer hardware and software, related research in the field of Elasto Hydrodynamic Lubrication (EHL) with non-smooth surfaces is increasing. In general the configuration to be considered is a two dimensional transient one, since the (rough) surfaces move with respect to the contact. This time dependent point contact problem has been studied experimentally by Kaneta and numerically by, amongst others, the authors. For a single feature a good correlation between experiments was observed, both quantitatively and qualitatively [31].

In the present paper the influence (transient and steady state) of a global surface feature, i.e. waviness, on pressure and film thickness for such a two dimensional contact is investigated for operating conditions identical to those used previously in experimental research. The transient results apply to transverse waviness which is confined to one surface. Film thickness and pressure distributions have been calculated as a function of time for different slide to roll ratios. As was previously observed for the infinitely wide line contact, it is shown that film thickness modulations tend to be propagated through high viscosity regions at the average surface speed. For the case of waviness this implies that the film profile in such a region will be a combination of two oscillations, i.e. one component with the wavelength of the undeformed waviness and the second with a wavelength that is larger or smaller. With increasing load the latter oscillation will tend to dominate the film thickness.

\section{INTRODUCTION}

Many years after the tribological society was asked the question whether or not engineering surfaces in concentrated contacts could be sucressfully lubricated, ensuring an "unlimited" life, the question has reappeared. To understand this renewed interest, it is necessary to reconsider the original answer. Successful lubrication was predicted whenever the calculated lubricant film thickness exceeded three times the combined roughness of the two "contacting" surfaces. Whatever happened with (much) thinner films was not specified!

Both technological and economical pressures now urge the tribological community to study what happens under such thin film conditions. These factors include, higher loads, higher operating temperatures, severely starved contacts (grease lubrication), and the high costs of large safety margins concerning size (weight), power consumption (viscosity) and surface roughness.

As a result, the current question is to predict the minimum film thickness required to obtain a certain operating life, under given operating conditions and with a given surface texture.

To answer this question much more (precise) information is required, not only with respect to the exact operating conditions and material properties, of both bulk and surface properties of the lubricant and the solids, but also a precise knowledge of the roughness topography.

It is not certain whether this problem, posed in its most general form, can be answered at all. Neither is it clear which approach will be the most fruitful in providing a (partial) answer. A possible approach is a straightforward extension of the existing models of Elasto Hydrodynamic Lubri- 
cation to include the kinematics and geometry of the problem of interest. This approach requires the solution of a number of subproblems:

- An efficient (fast) and stable numerical method to solve the transient equations with sufficient detail.

- A thorough understanding of the lubricated contact behaviour under realistically starved conditions.

- An extension of the model to incorporate local rheological and thermal behaviour.

- A response to whether or not such a model can use the Reynolds equation with its integration over the height of the film.

- And finally, the model needs to include surface or interfacial behaviour, where it is different from the bulk behaviour.

With the advances in numerical solution techniques and the general availability of powerful computers, the first step towards a more complete answer of this problem seems feasible: the transient EHL point contact with moving wavy surfaces can now be studied. Such an investigation is of interest as a prelude to the study of Elasto Hydrodynamic Lubrication of general non-smooth surfaces. Even in its own right the transient problem of EHL with wavy surfaces is of importance, because experiments using optical interferometry, can provide detailed information concerning the film thickness as a function of space and time. As such, this problem is very suited to compare film thickness calculations to film thickness measurements, and to investigate the validity of the utilized viscosity pressure relations and ultimately of the Reynolds equation itself.

A combination of several factors makes that this problem remains difficult to solve numerically. First of all, a spatial resolution is required which surpasses the one needed for the equivalent smooth contact case. Secondly, the two dimensional character requires the use of (many) discrete points in two dimensions. Thirdly, the waviness requires a solution method with a stability well beyond the one necessary to solve the stationary smooth problem. And finally, a time increment which should be comparable to the spatial resolution adds a third dimension to the problem, multiplies the solution time and requires even stricter supervision of the (propagation of) numerical errors.

The combination of these difficulties has inspired a number of investigators to study a simpler problem first such as the stationary oneor two dimensional problem: $[11,12,17-21,27,29]$. The transient one dimensional case was studied in: $[6-9,13,22-25,28,30]$, and the transient two dimensional problem was addressed in $[1-3,31]$. Experimental results are reported in [14-16,32].

As was shown in the theoretical paper [30] the solution of the general non-smooth surface contact problem requires a transient solution of the EHL equations. In that paper as well as in the present one the authors have selected Multigrid solvers, since they meet the two requirements necessary to tackle this particular problem; they are fast and robust.

\subsection{Notation}

$A$ amplitude

$\mathcal{A}$ dimensionless amplitude, $\mathcal{A}=A R_{x} / b^{2}$

$b$ radius of Hertzian contact, $b=\sqrt[3]{\left(3 w R_{x}\right) /\left(2 E^{\prime}\right)}$

$E \quad$ modulus of elasticity

$E^{\prime} \quad$ reduced modulus of elasticity,

$$
2 / E^{\prime}=\left(1-\nu_{1}^{2}\right) / E_{1}+\left(1-\nu_{2}^{2}\right) / E_{2}
$$

$G$ dimensionless materials parameter,

$$
G=\alpha E^{\prime}
$$

$h \quad$ film thickness

$h_{\min }$ minimum film thickness

$H$ dimensionless film thickness, $H=h R_{x} / b^{2}$

$H_{\text {min }}$ dimensionless minimum film thickness,

$$
H_{\min }=h_{\min } R_{x} / b^{2}
$$

$H_{0} \quad$ integration constant

$\mathcal{I} \quad$ intensity in pseudo interference graph

$L$ dimensionless material parameter (Moes), $L=G(2 U)^{0.25}$

$M$ dimensionless load parameter (Moes), $M=W(2 U)^{-0.75}$

$n_{x}, n_{y}$ number of points in $x$ and $y$

$p \quad$ pressure

$p_{h}$ maximum Hertzian pressure, $p_{h}=(3 w) /\left(2 b^{2}\right)$

$P$ dimensionless pressure, $P=p / p_{h}$ 
$K_{x} \quad$ reduced radius of curvature in $x$ direction, $1 / R_{x}=1 / R_{x 1}+1 / R_{x 2}$

$R_{y} \quad$ reduced radius of curvature in $y$ direction, $1 / R_{y}=1 / R_{y 1}+1 / R_{y 2}$

$R$ dimensionless deviations from the smooth profile

t. time

$T$ dimensionless time, $T=\left(t u_{s}\right) /(2 b)$

$u_{1} \quad$ velocity of lower surface (smooth)

$u_{2} \quad$ velocity of upper surface (wavy)

$u_{s} \quad$ sum velocity, $u_{s}=u_{1}+u_{2}$

l' dimensionless speed parameter,

$U=\eta_{0} u_{s} /\left(2 E^{\prime} R_{x}\right)$

$u r$ velocity ratio, $u r=u_{2} / u_{\text {s }}$

w external load per unit width

IV dimensionless load parameter, $W=w /\left(E^{\prime} R_{x}^{2}\right)$

w wavelength of surface feature

$\mathcal{W}$ dimensionless wavelength, $\mathcal{W}=\omega / b$

$x, y$ coordinates

$x_{i}$ location of surface feature

$x: \quad$ location of surface feature at $t=0$

$X, Y$ dimensionless coordinates, $X=x / b, Y=y / b$

$X_{d}$ dimensionless location of surface feature, $X_{d}=x_{d} / b$

$X_{s}$ dimensionless location of surface feature at $T=0, X_{s}=x_{s} / b$

$X_{a}, X_{b}$ dimensionless boundaries of the domain $X_{a}=x_{a} / b, X_{b}=x_{b} / b$

$Y_{a}, Y_{b}$ dimensionless boundaries of the domain $Y_{a}=y_{a} / b, Y_{b}=y_{b} / b$

$\therefore \quad$ viscosity index (Roelands equation)

a pressure viscosity index

a dimensionless parameter, $\bar{\alpha}=\alpha p_{h}$

$\Delta_{T} \quad$ dimensionless time increment

$\Delta_{X} \quad$ dimensionless space increment

c coefficient in Reynolds equation, $\epsilon=\left(\bar{\rho} H^{3}\right) /(\bar{\eta} \lambda)$

$\lambda$ dimensionless speed parameter, $\lambda=\left(6 \eta_{0} u_{s} R_{x}^{2}\right) /\left(b^{3} p_{h}\right)$

$\eta \quad$ viscosity

$\eta_{0} \quad$ viscosity at ambient pressure

$\eta \quad$ dimensionless viscosity, $\bar{\eta}=\eta / \eta_{0}$

p density

$\rho_{0} \quad$ density at ambient pressure

$\rho \quad$ dimensionless density, $\bar{\rho}=\rho / \rho_{0}$

$\Sigma \quad$ slide to roll ratio, $\Sigma=2\left(u_{1}-u_{2}\right) /\left(u_{1}+u_{2}\right)$

\section{THEORY}

For completeness this section first presents the equations to be solved. Subsequently, the physical parameters describing the contact conditions are given.

\subsection{Equations}

The two dimensional transient Reynolds equation is written in a dimensionless form:

$$
\begin{array}{r}
\frac{\partial}{\partial X}\left(\epsilon \frac{\partial P}{\partial X}\right)+\frac{\partial}{\partial Y}\left(\epsilon \frac{\partial P}{\partial Y}\right) \\
-\frac{\partial(\bar{\rho} H)}{\partial X}-\frac{\partial(\bar{\rho} H)}{\partial T}=0
\end{array}
$$

The $X$-coordinate is chosen to coincide wih the rolling direction. The boundary conditions are $P\left(X_{a}, Y, T\right)=P\left(X_{b}, Y, T\right)=P\left(X, Y_{a}, T\right)=$ $P\left(X, Y_{b}, T\right)=0, \forall X, Y, T$ where $X_{a}, X_{b}, Y_{a}$ and $Y_{b}$ denote the boundaries of the domain. Furthermore, the cavitation condition $P(X, Y, T) \geq 0$, $\forall X, Y, T$ must be satisfied throughout the domain. $\epsilon$ is defined according to:

$$
\epsilon=\frac{\bar{\rho} H^{3}}{\bar{\eta} \lambda} \quad \text { with } \quad \lambda=\frac{6 \eta_{0} u_{s} R_{x}^{2}}{b^{3} p_{h}}
$$

The density $\rho$ is assumed to depend on the pressure according to the Dowson and Higginson relation [10] and the Roelands viscosity pressure relation [26] is used. The film thickness equation is made dimensionless using the same parameters and accounting for a moving surface feature reads:

$$
\begin{aligned}
H(X, Y, T)= & H_{0}(T)-\mathcal{R}(X, Y, T)+\frac{X^{2}}{2}+\frac{Y^{2}}{2} \\
& -\frac{1}{\pi} \int_{\Omega} \frac{P\left(X^{\prime}, Y^{\prime}, T\right) d X^{\prime} d Y^{\prime}}{\sqrt{\left(X-X^{\prime}\right)^{2}+\left(Y-Y^{\prime}\right)^{2}}}
\end{aligned}
$$

where $H_{0}(T)$ is an integration constant, and $\mathcal{R}(X, Y, T)$ denotes the undeformed geometry of the upper body at dimensionless time $T . \mathcal{R}_{t}$ stands for a transverse waviness and $\mathcal{R}_{l}$ denotes longitudinal waviness. Note that $\mathcal{R}_{l}(X, Y, T)$ does not vary with $X$ nor with $T$.

$\mathcal{R}_{t}(X, Y, T)= \begin{cases}0, & X \geq X_{d} \\ \mathcal{A} \sin \left(2 \pi \frac{X_{d}-X}{\mathcal{W}}\right), & X<X_{d}\end{cases}$ 
$\mathcal{R}_{l}(X, Y, T)=\mathcal{A} \cos \left(2 \pi \frac{Y}{\mathcal{W}}\right)$

$X_{d}=X_{s}+2 u_{2} / u_{s} T$

In this paper $\mathcal{W}=0.32$ and $\mathcal{A}=0.045$ will be used.

At all times the force balance condition is imposed, i.e. the integral over the pressure must balance the externally applied contact load. This condition determines the value of the integration constant $H_{0}(T)$ in equation (2). Expressed in the dimensionless variables the force balance equation reads:

$\int_{\Omega} P(X, Y, T) d X d Y-\frac{2 \pi}{3}=0 \quad \forall T$

\begin{tabular}{|l|l|l|}
\hline Parameter & Value & Dimension \\
\hline$E^{\prime}$ & $1.17 \times 10^{11}$ & {$[\mathrm{~Pa}]$} \\
\hline$\alpha$ & $2.2 \times 10^{-8}$ & {$\left[\mathrm{~Pa}^{-1}\right]$} \\
\hline$\eta_{0}$ & 1.22 & {$[\mathrm{Pas}]$} \\
\hline$u_{s}$ & $9.6 \times 10^{-2}$ & {$\left[\mathrm{~m} \mathrm{~s}^{-1}\right]$} \\
\hline$R_{x}$ & $1.27 \times 10^{-2}$ & {$[\mathrm{~m}]$} \\
\hline$b$ & $1.84 \times 10^{-4}$ & {$[\mathrm{~m}]$} \\
\hline$p_{h}$ & $0.54 \times 10^{9}$ & {$[\mathrm{~Pa}]$} \\
\hline$h_{\text {min,smooth }}$ & 0.21 & {$[\mu \mathrm{m}]$} \\
\hline$h_{\text {cen, smooth }}$ & 0.40 & {$[\mu \mathrm{m}]$} \\
\hline \hline \multicolumn{3}{|c|}{ Dimensionless parameters } \\
\hline$M$ & 77 & \\
\hline$L$ & 7.9 & \\
\hline \hline $\bar{\alpha}$ & 12.1 & \\
\hline$\lambda$ & $3.4 \times 10^{-2}$ & \\
\hline \hline$W$ & $2.0 \times 10^{-6}$ & \\
\hline$U$ & $3.9 \times 10^{-11}$ & \\
\hline$G$ & $2.63 \times 10^{3}$ & \\
\hline
\end{tabular}

Table 1 Different parameters and their values for the presented loading condition.

\subsection{Conditions}

The parameters used to describe the contact conditions in the calculations are given in Table 1 , together with some dimensionless parameters. 'The numerical parameters used in the calculation are given in Table 2 . When compared to

\begin{tabular}{|l|l|}
\hline Parameter & Value \\
\hline$X_{a}$ & -2.5 \\
\hline$X_{b}$ & 1.5 \\
\hline$Y_{a}$ & -2.0 \\
\hline$Y_{b}$ & 2.0 \\
\hline$\Delta_{T}$ & $0.015625 /-$ \\
\hline$\Delta_{X}$ & $1.5625 \times 10^{-2} / 7.8125 \times 10^{-3}$ \\
\hline$n_{x} * n_{y}$ & $257 * 257 / 513 * 513$ \\
\hline
\end{tabular}

Table 2 Different parameters in the numerical simulation (transient/stationary).

the contact conditions considered before, i.e. in [31] the maximum Hertzian pressure is the same but the sum velocity is about 4 times larger.

\section{NUMERICAL SIMULATION}

Second order discretisation of the equations with respect to time was combined with double discretisation to obtain second order accuracy in the spatial variables $X, Y$. Multilevel FAS was used to obtain fast convergence of the solution (at each time step) to a numerical error below the (incremental) discretisation error, while Multilevel integration for the fast calculation of the elastic deformation integrals was employed.

The general theory of Multigrid solvers is found in for instance $[4,5]$, applications to EHL are described in $[20,29]$, and in the references of these works. Details of the numerical solution of the transient problem can be found in $[30,31]$.

For the transient solutions the calculational domain was $-2.5 \leq X \leq 1.5,-2.0 \leq Y \leq 2.0$ and the finest grid in the multilevel cycle (level 8) consisted of $257 * 257$ points. 'This corresponds to a mesh size $\Delta X=\Delta Y=0.015625$, the time increment was $\Delta T=0.015625$. The simulation was started with the location of the waviness at $X_{s}=X_{d}(T=0)=-2.0$, in order to start the computation with an undisturbed (smooth surface) solution. It is important to choose this location sufficiently far in the inlet to ensure that possible numerical start-up effects have died out by the time the waviness really starts to affect the solution of pressure and film thickness. The stationary problem was solved using the same domain but the calculations were executed employ- 
ing a finest grid with $\Delta X=\Delta Y=0.0078125$, and $513 * 513$ points (level 9 ).

Table 2 lists the numerical parameters used in the calculations.

To facilitate the comparison with the interferometry measurements, the film thickness results are presented using pseudo interference graphs. These pseudo interference graphs were plotted using the intensity $\mathcal{I}$ (defined by equation (7)) where $\mathcal{I}=1$ produces a white square of $\Delta_{X} * \Delta_{Y}$, $\mathcal{I}=0$ a black square, and grey squares for $0<\mathcal{I}<1$. The area plotted extends from $-1.5<X<1.5$ and $-1.5<Y<1.5$.

$I(X, Y, T)=0.5+0.5 \cos \left(\frac{2 \pi H(X, Y, T)}{\Lambda}\right)$

where: $\Lambda=$ dimensionless wavelength, $\Lambda=0.05$.

In Figure 1 the smooth surface case is depicted: the pseudo interference plot of the film thickness, and pressure and film thickness profiles for $Y=0$ and $X=0$ respectively. This figure displays all classical features of a medium load EHL contact: constant film thickness in the central (high pressure) region, horse shoe shaped restriction with a minimum film thickness occuring near $X=0,|Y|=1$.

\section{STATIONARY RESULTS}

Figure 2 displays the stationary transverse waviness results. As has been shown before, the waviness is almost completely deformed in the high-pressure region, because of the near absence of pressure induced (Poiseuille) flow; the flow is Couette dominated. This flattening of the waviness requires large pressure ripples. The global film thickness depends on whether the waviness causes an increased flow in the inlet, or whether it decreases the lubricant flow into the contact. In the side lobe region, where the pressures are lower, the original waviness shape is superimposed upon the horse-shoe shape.
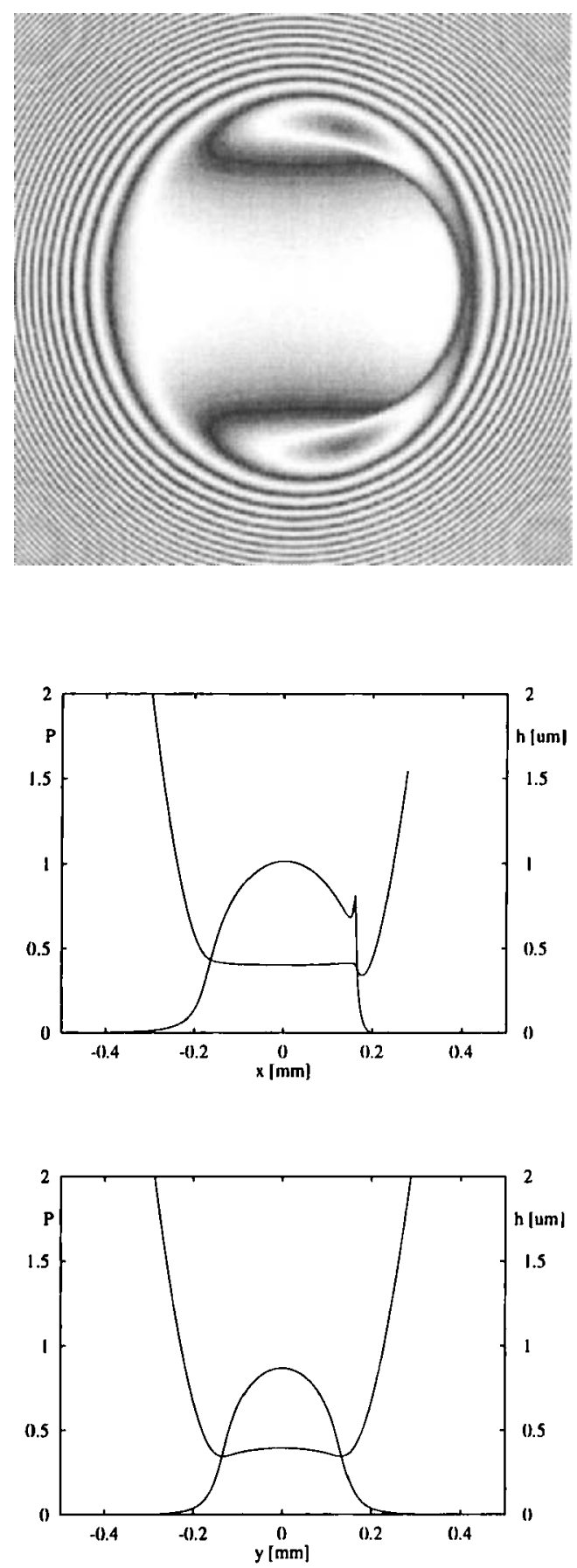

Figure 1. Pseudo interference plot $H$ (top) and pressure and film thickness for $Y=0$ (middle) and for $X=0$ (bottom), smooth surface. 

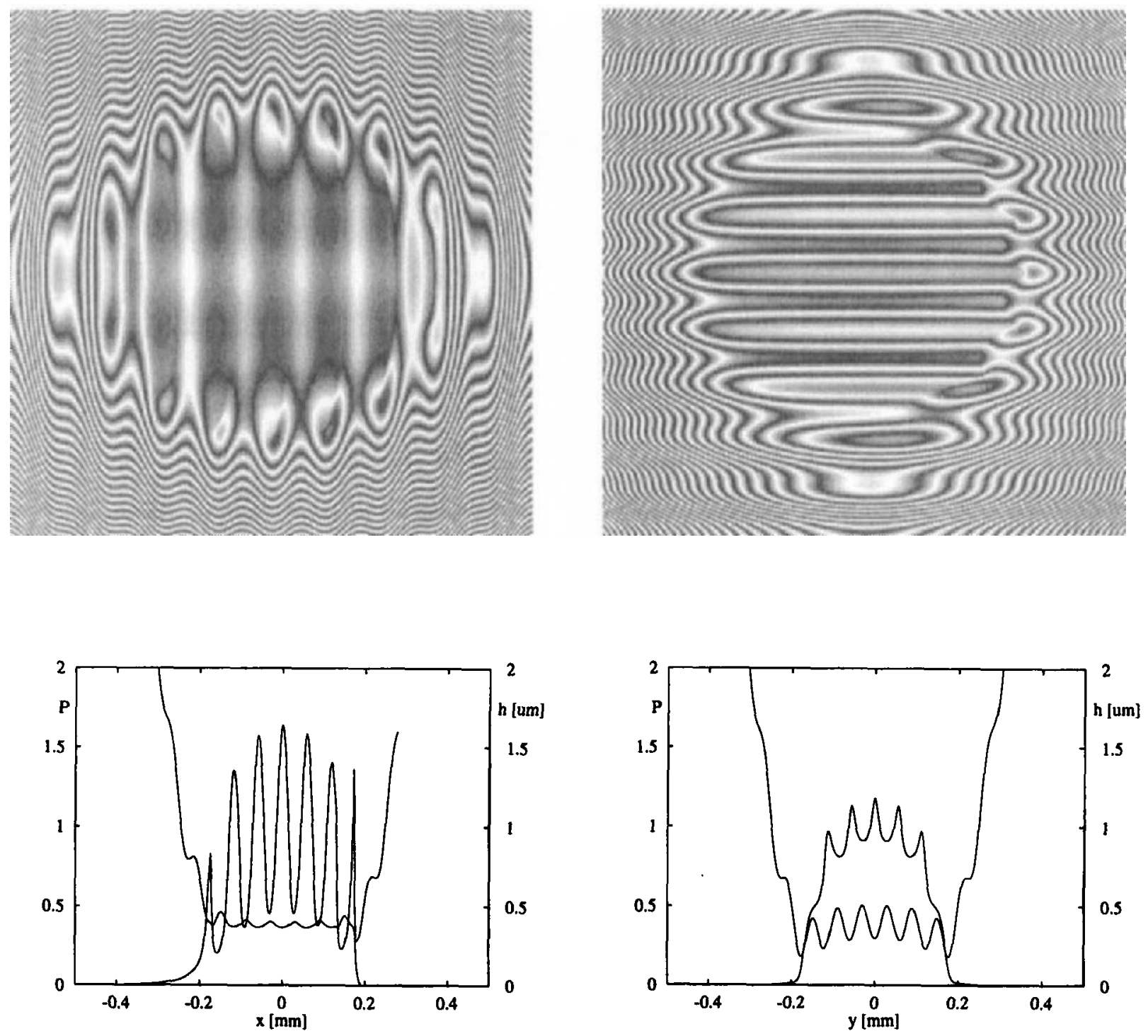

Figure 2. Pseudo interference plot $H$ (top) and pressure and film thickness for $Y=0$ (bottom), transverse waviness.

Figure 3. Pseudo interference plot $H$ (top) and pressure and film thickness for $X=0$ (bottom), longitudinal waviness. 

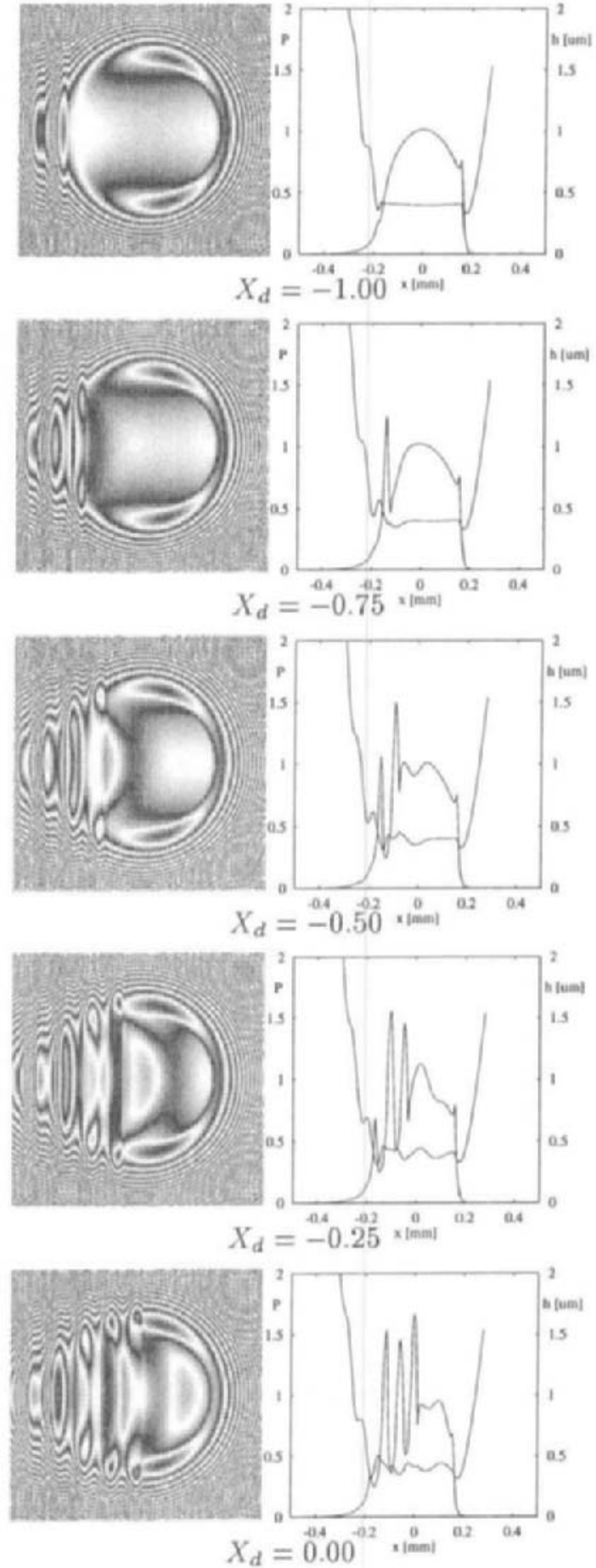
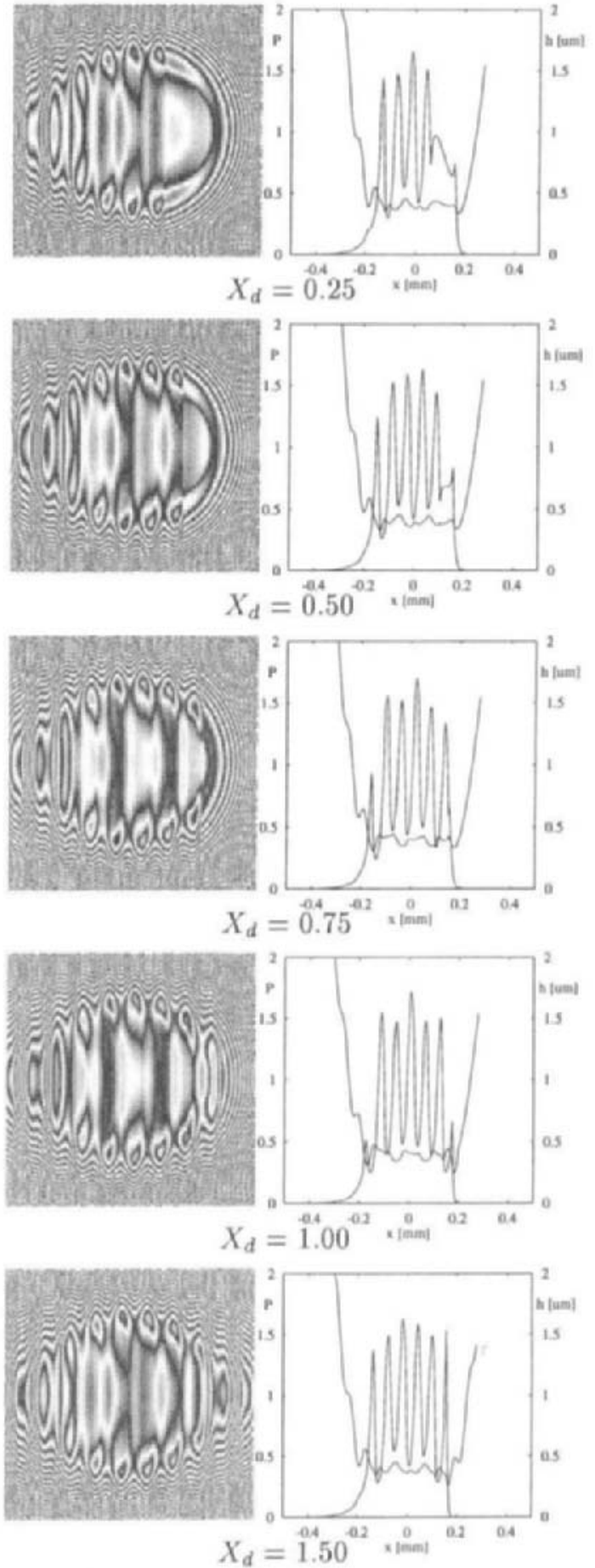

Figure 4: $u r=0.25:$ pseudo interference plot $H$ (left) and pressure and film thickness on line $Y=0$ (right), for different positions $X_{d}$ 

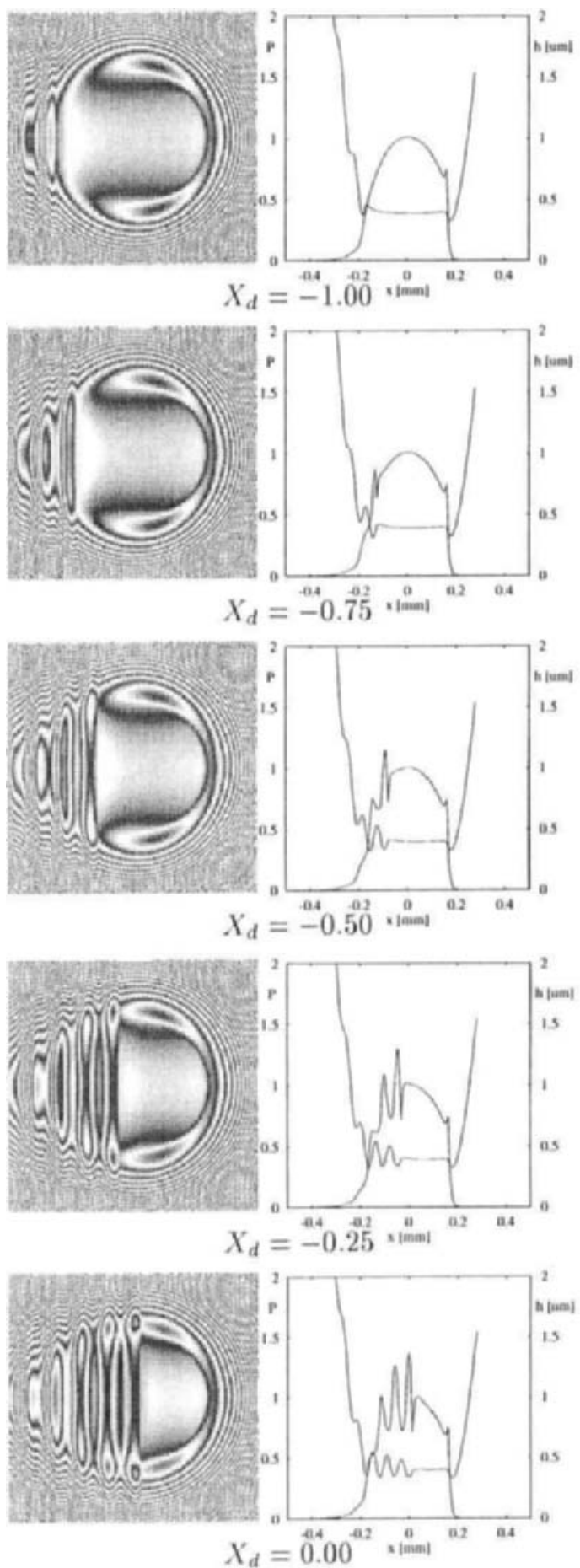
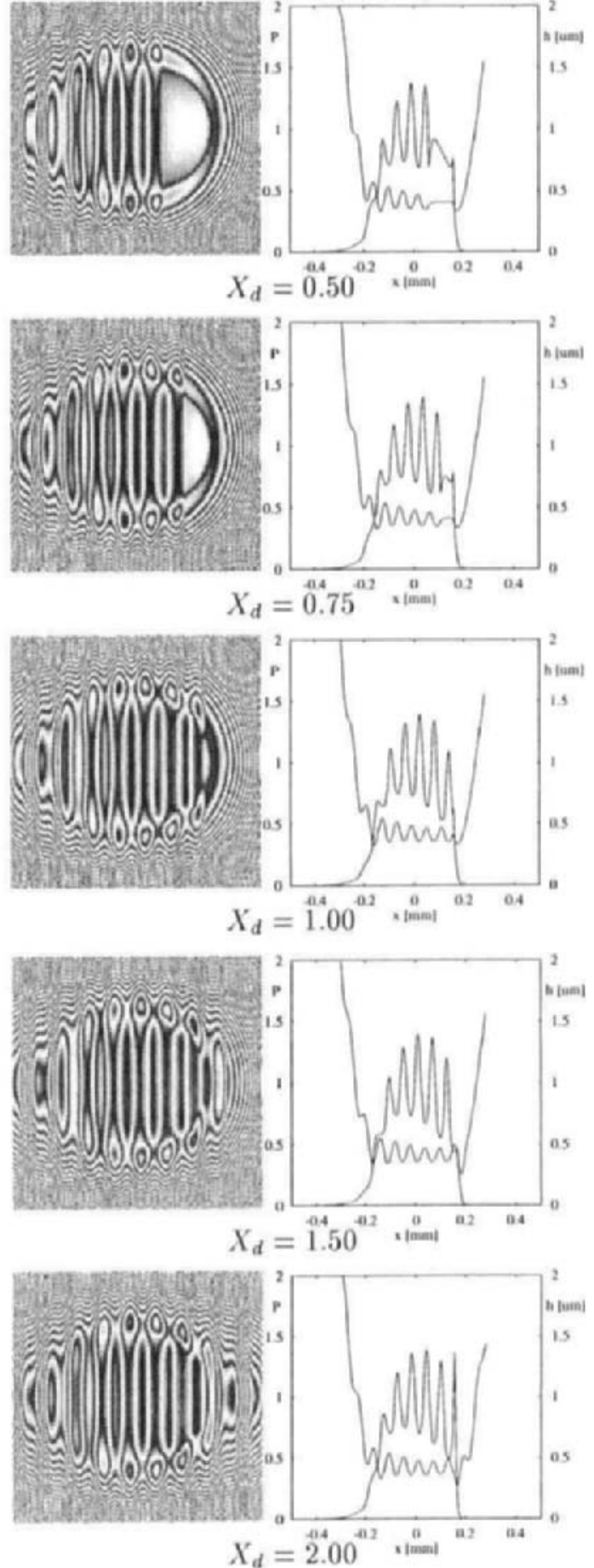

Figure 5: $u r=0.50:$ pseudo interference plot $H$ (left) and pressure and film thickness on line $Y=0$ (right), for different positions $X_{d}$ 

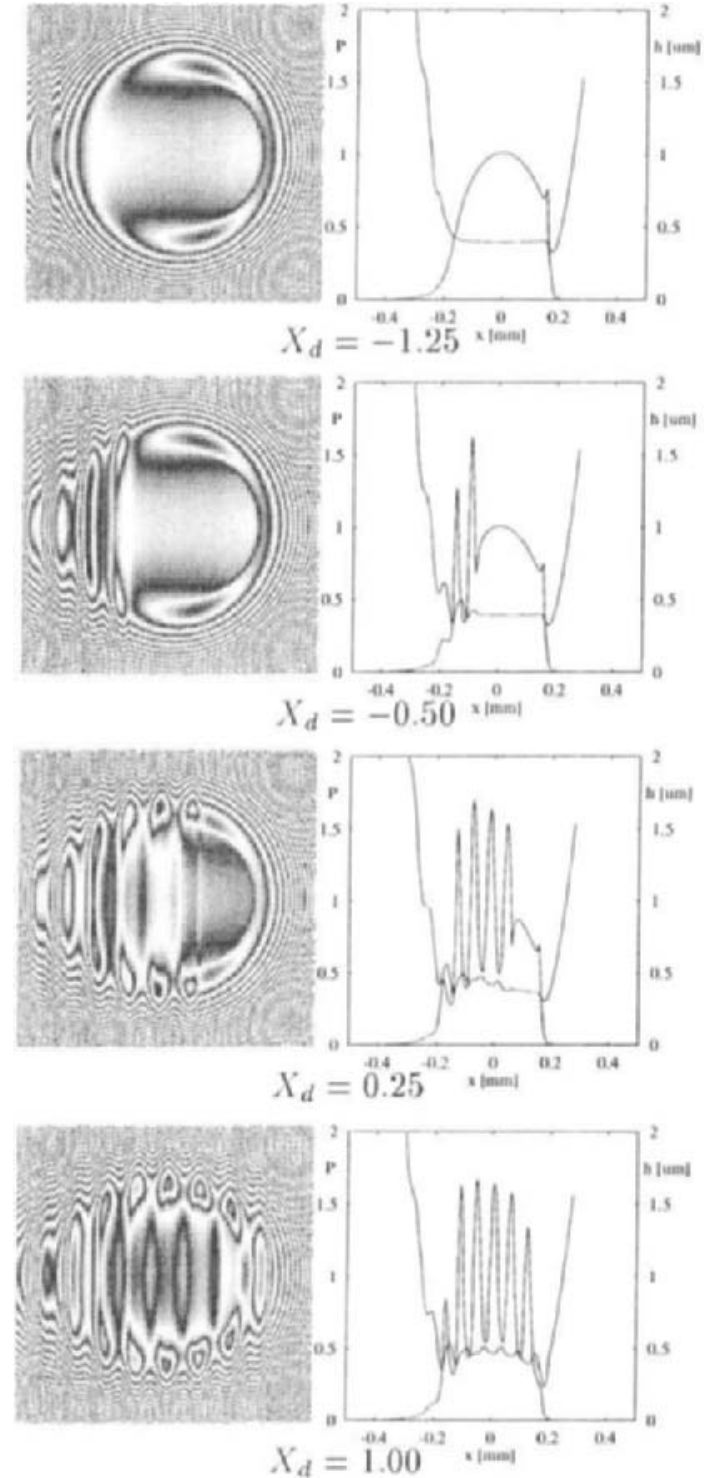
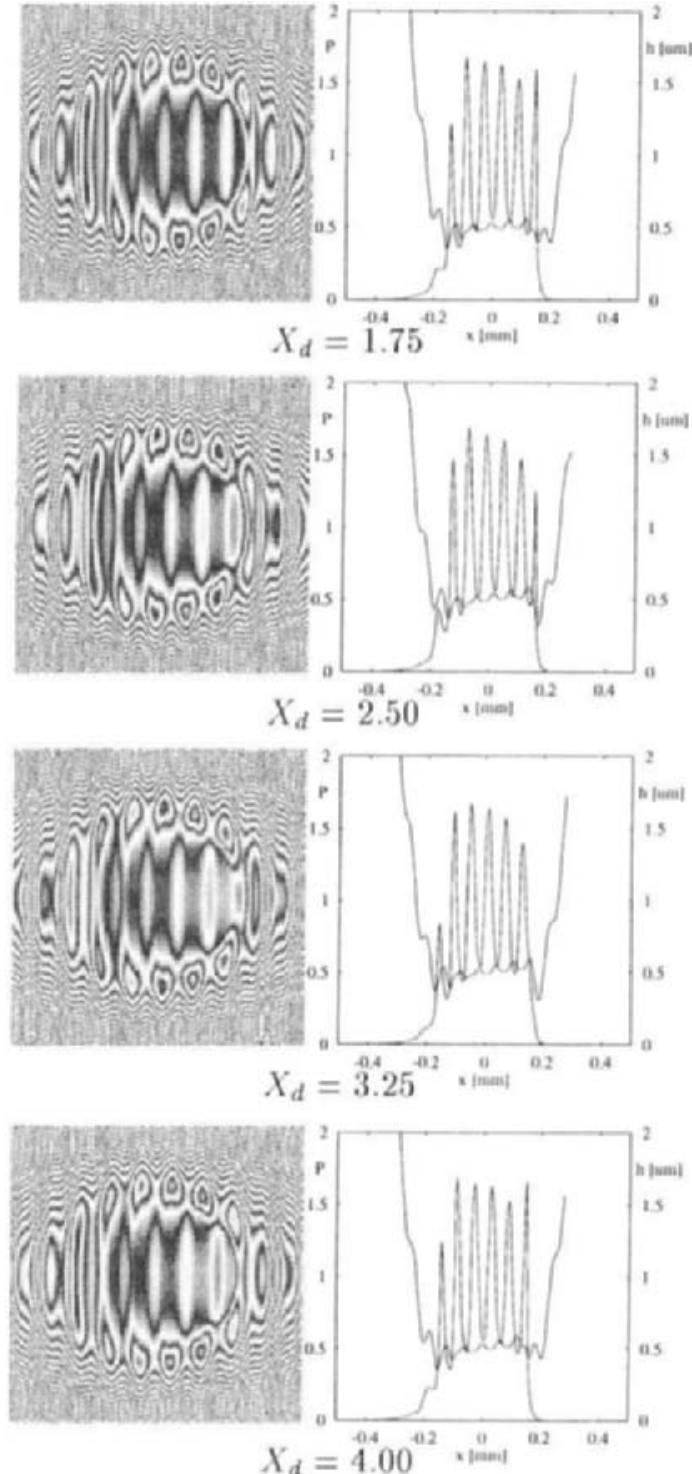

Figure 6: pseudo interference plot $H$ (left) and pressure and film thickness on line $Y=0$ (right), for different positions $X_{d}$. 
Figure 3 displays the longitudinal waviness results. For this particular orientation the waviness deforms very little, and the pressure undulations are relatively small. The deformation is that small because the lubricant flux into the contact is already (correctly) modulated by the waviness in the inlet region. This contrary to the case in which the waviness is at a certain angle with respect to the surface velocity, see [3].

\section{TRANSIENT RESULTS}

Obviously the case of longitudinal roughness remains a steady state problem, even if the rough surface moves. However, in the case of transverse waviness the results will depend on the velocity of the rough surface compared to the average velocity. In this section transient results of the transverse waviness moving through the contact are presented. The waviness is located on the upper surface and consequently moves with the velocity $u_{2}$.

\section{1. $\mathrm{ur}=0.25, \Sigma=1$}

Figure 4 displays the waviness results for a surface speed ratio $u r=u_{2} / u_{s}=0.25$. These 10 figures show through pseudo interference graphs and central line pressure and film thickness profiles, the progression of the waviness through the contact, at locations $X_{d}=-1.0$ to 1.5. As the wavy surface has a velocity lower than the average surface speed (= lubricant propagation speed) the waviness influence progresses in front of the waviness itself. This causes an important film thickness disturbance and a small pressure disturbance to advance ahead of the waviness position. Pressure and film thickness disturbances are thus out of phase. From $X_{d}=1.0$ onwards the fluctuations become periodic, with a period of approximately $5 / 8$, see Figure 7 . 'This figure displays the minimum and central $(X=Y=0)$ film thickness as a function of time. The central film thickness oscillates around its smooth surface value, whereas the minimum film thickness decreases by $20 \%$ and has a relatively constant value. Note the delay between the time when $H_{c e n}$ and $H_{m i n}$ are affected by the waviness. $H_{\min }$ occurs in the low pressure region, hence the wave propagation speed is given by $u_{p}=u_{s} / 4$, whereas $H_{c e n}$ is located in the high pressure region where $u_{p}=u_{s} / 2$.

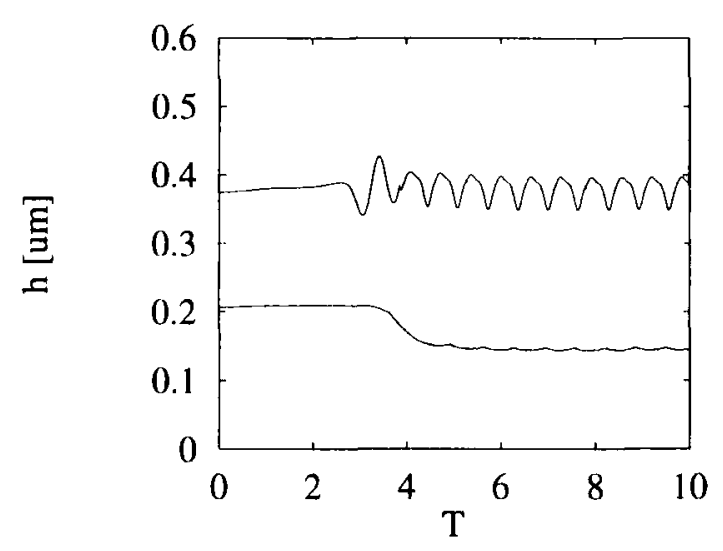

Figure 7. Central and minimum film thickness as a function of time, for $u r=0.25$.

\section{2. $\mathrm{ur}=0.50, \Sigma=0$}

Figure 5 displays the waviness results for a surface speed ratio $u r=u_{2} / u_{s}=0.50$. These $10 \mathrm{fig}$ ures show the progression of the waviness through the contact, at locations $X_{d}=-1.0$ to 2.0 . As both surfaces have identical velocities (equal to the lubricant velocity) pressure and film thickness disturbances move at the same speed, and remain in phase. From $X_{d}=1.0$ onwards the fluctuations become periodic, see Figure 8 . The central film thickness oscillates around an average value which is almost $10 \%$ larger than its smooth surface value, whereas the minimum film thickness decreases by $30 \%$ and varies slightly. The fluctuations in $H_{\min }$ and $H_{\text {cenn }}$ start simultaneously and both have a period of approximately $5 / 16$.

\section{3. $u r=0.75, \Sigma=-1$}

Figure 6 displays the waviness results for a surface speed ratio $u r=u_{2} / u_{s}=0.75$. These 8 figures show the progression of the waviness through the contact, at locations $X_{d}=-1.25$ to 4.0 . As the wavy surface has a velocity larger than the average surfaces speed, film thickness disturbances 


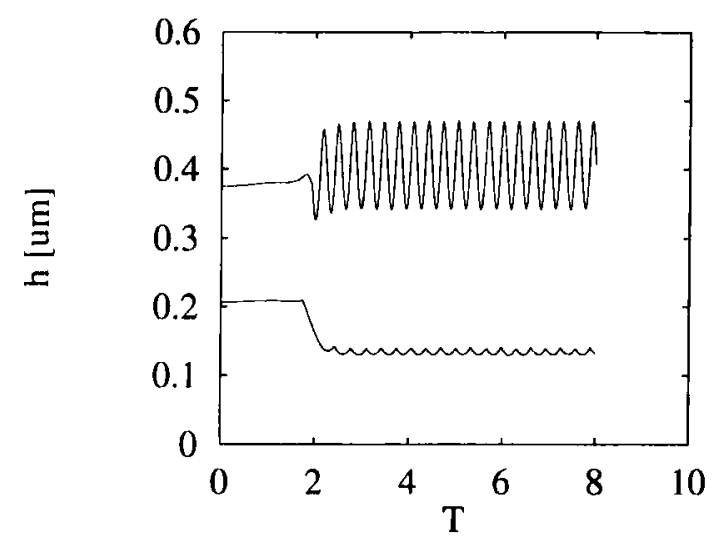

Figure 8. Central and minimum film thickness as a function of time, for $u r=0.50$.

trail behind the location of the roughness, and the pressure fluctuations. Pressure and film thickness fluctuation are thus out of phase. From $X_{d}=1.0$ onwards they become periodic, with a period of approximately $5 / 24$, see Figure 9 . The central film thickness oscillates around an average value which is $30 \%$ larger than the smooth film value, the minimum film thickness oscillates around a value almost $20 \%$ smaller than the smooth film vilue.

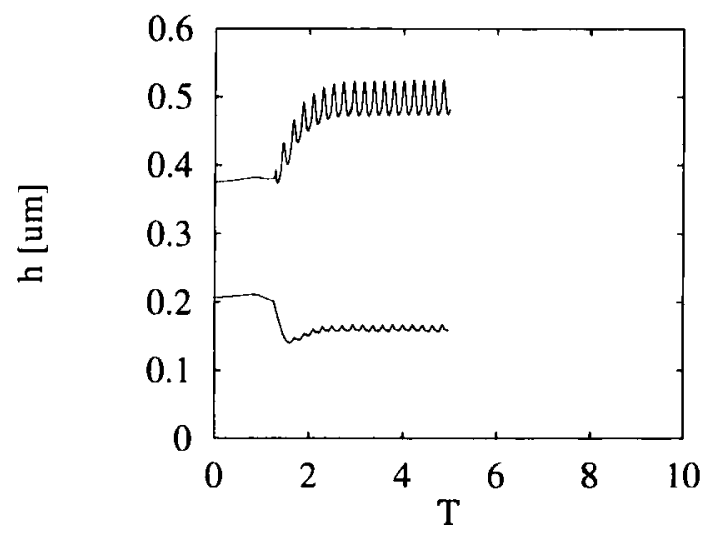

Figure 9. Central and minimum film thickness as a function of time, for $u r=0.75$.

\section{NUMERICAL ACCURACY}

The stationary cases were calculated on a grid of $513 * 513$ points, $\Delta_{X}=0.0078125$. The transient calculations were performed on a grid of $257 * 257$ points, $\Delta_{X}=0.015625$, with a time step $\Delta_{T}=0.015625$. The initial position of the start of the waviness was $X_{s}=X_{d}(T=0)=-2.0$.

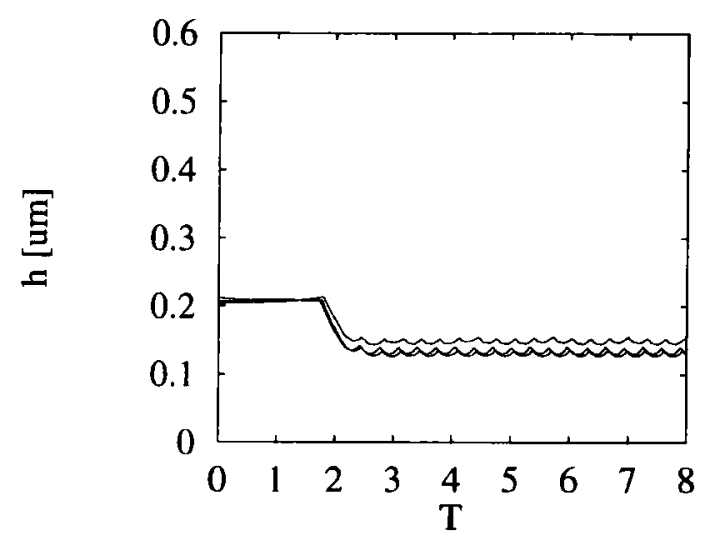

Figure 10. Minimum film thickness for $u r=0.5$ as a function of time, second order discretisation on levels 7 and 8 , first order discretisation on level 8.

The stationary results have a film thickness error well below $1 \%$, see [31]. The transient results are less accurate, for two reasons. First of all they have a mesh size twice as large, secondly because the total error accumulates over time. Figures 10 and 11 show the difference in minimum and central film thickness as a function of time, for second order discretisation on level 7 and 8 and first order on level 8 . From these figures it can be concluded that the second order error on level 8 , in the minimum film thickness is of the order of $5 \%$, the error in the central film thickness is much larger. The error in the first order scheme on level 8 is, somewhat surprisingly, smaller than the second 


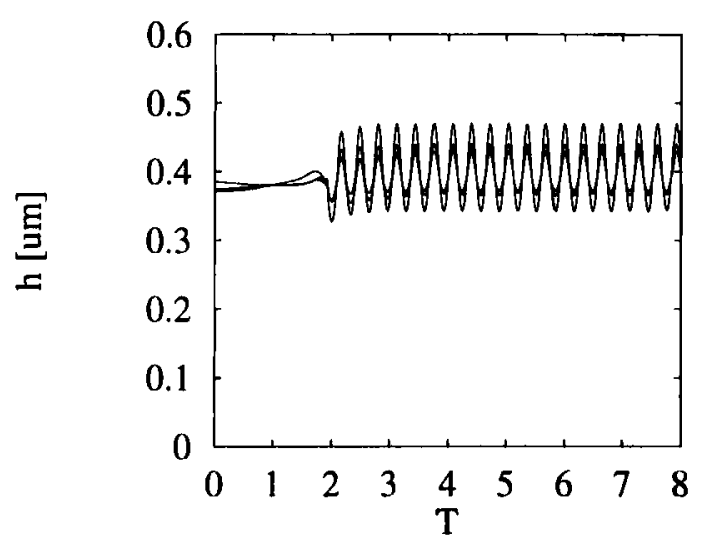

Figure 11. Central film thickness for $u r=0.5$ as a function of time, second order discretisation on levels 7 and 8 , first order discretisation on level 8 .

order level 7 error. The reduction in the waviness amplitude in Figure 5 is another expression of this discretisation error (artificial damping).

\section{DISCUSSION}

Whereas the agreement between the experimentally obtained film thickness profiles [15] and the numerical ones [30] was both qualitatively and quantitatively very good, for a single feature, the continuous waviness has not shown a similar agreement. First of all it was not possible to numerically reproduce Kaneta's low speed experimental conditions [16], $U=9 \times 10^{-12}$, because negative film thicknesses were obtained. Secondly, the waviness amplitude in the calculations was only half the value used in [16]. From Figure 4 and 6 it can be observed that the pressure variations have an amplitude of roughly $p_{h} / 2$, hence doubling the waviness would result in local cavitation, which the current algorithm cannot cope with. Hence, the authors think that in the experiments the flow is "reset" between the waviness crests, because the low pressure allows the Poiseuille component to compete with the Cou- ette flow, locally inside the contact. As a result the experimental pressure and film thickness cannot develop independently, and thus cannot show the phase difference calculated numerically.

\section{CONCLUSION}

This paper has studied in detail the pressure and film thickness variations in a transient two dimensional circular contact with longitudinal and transverse waviness.

For the transient waviness results, the case of pure rolling $u r=0.5$ gives the smallest minimum film thickness, and by far the largest central film thickness variations. Because sliding is completely absent, no additional lubricant moves over, and flattens (averages) the waviness. This does not imply that the pure rolling condition is therefore the most dangerous with respect to failure. The energy dissipation in any contact depends strongly on the surface speed difference, which is zero in the case of pure rolling, and the maximum pressure is also the smallest.

A comparison of Figures 7 and 9 shows an interesting phenomenon. The periodic variations of $H_{\text {cen }}$ for $u r=0.75$ as a function of $T$ are the inverse of the ones for $u r=0.25$ albeit at a different time scale. A comparison of Figures 4 and 6 shows that the film thickness profiles as a function of $X$ are completely different. This indicates that this film thickness is caused by a combination of several waves propagating through the contact at different speeds, as explained by Greenwood and Morales Espejel [13,24].

More precise information about these two waves comes from a closer examination of Figure 7 . It shows the start of a harmonic variation in $H_{c e n}$ around $T=2.5$. This is the induced wave, that is propagating through the contact at $u_{p}=u_{s} / 2$. At approximately $T=3.5$ a second wave arrives at $X=0$ and interferes with the the variation in $H_{c e n}$. This wave has a propagation speed of $u_{p}=u_{2}=u_{s} / 4$ and is caused by the waviness geometry itself. Contrary to the incuced wave, this wave also exists in the low pressure region, as is indicated by the variations in $H_{m i n}$, which also start at $T=3.5$. 


\section{ACKNOWLEDGMENT}

Part of this research was supported by a fellowship of the "Royal Netherlands Academy of Arts and Sciences" and by the Feinberg Graduate School of the Weizmann Institute of Science, Israel.

\section{REFERENCES}

1. Ai, X., Cheng, H.S., and Zheng, L., 1993, "A Transient Model for MicroElastohydrodynamic Lubrication with TreeDimensional Irregularities," ASME JOT, 115, pp. 102-110.

2. Ai, X., and Cheng, H.S., 1994, "The Influence of Moving Dent on Point EHL Contacts," STLE Trib. Trans., 37, pp. 323-335.

3. Ai, X., and Cheng, H.S., "The Effects of Surface Texture on EHL Point Contacts," submitted to ASME JOT.

4. Brandt, A., 1984, "Multigrid Techniques: 1984 Guide with Applications to Fluid Dynamics", GMD-Studien 85.

5. Briggs, W.L., 1987, "A Multigrid Tutorial", SIAM.

6. Chang, L., Cusano, C., and Conry, T.F., 1989, "Effects of Lubrication Rheology and Kinematic Conditions on MicroElastohydrodynamic Lubrication," ASME JOT, 111, pp. 344-351.

7. Chang, L., and Webster, M.N., 1991, "A Study of Elastohydrodynamic Lubrication of Rough Surfaces," ASME JOT, 113, pp. 110115.

8. Chang, L., 1992, "Traction in Thermal Elastohydrodynamic Lubrication of Rough Surfaces," ASME JOT, 114, pp. 186-191.

9. Chang, L., Webster, M.N., and Jackson, A., 1993, "On the Pressure Rippling in Elastohydrodynamic Lubrication of Rough Surfaces," ASME JOT, 115, pp. 439-444.

10. Dowson, D., and Higginson, G.R., 1966, "Elastohydrodynamic Lubrication, The Fundamentals of Roller and Gear Lubrication," Pergamon Press, Oxford, Great Britain.
11. Goglia, P.R., Cusano, C., and Conry, T.F., 1984, "The Effects of Surface Irregularities on the Elastohydrodynamic Lubrication of Sliding Line Contacts. Part I - Single Irregularities," ASME JOT, 106, pp. 104-112.

12. Goglia, P.R., Cusano, C., and Conry, T.F., 1984, "The Effects of Surface Irregularities on the Elastohydrodynamic Lubrication of Sliding Line Contacts. Part II - Wavy Surfaces," ASME JOT, 106, pp. 113-119.

13. Greenwood, J.A., and Johnson, K.L., 1992, "The Behaviour of Transverse Roughness in Sliding Elastohydrodynamically Lubricated Contacts," WEAR, 153, pp. 107117.

14. Kaneta, M., 1992, "Effects of Surface Roughness in Elastohydrodynamic Lubrication," JSME, III, 35, 4, pp. 535-546.

15. Kaneta, M., Sakai, T., and Nishikawa, H., 1992, "Optical Interferometric Observations of the Effects of a Bump on Point Contact EHL," ASME JOT, 114, pp. 779-784.

16. Kaneta, M., Sakai, T., and Nishikawa, H., 1993, "Effects of Surface Roughness on Point Contact EHL," STLE Trib. Trans., 36,4, pp. 605-612.

17. Kweh, C.C., Evans, H.P., and Snidle, R.W., 1989, "Micro-Elastohydrodynamic Lubrication of an Elliptical Contact with Transverse and Three-Dimensional Roughness," ASME JOT, 111, pp. 577-583.

18. Lee, R.T., and Hamrock, B.J., 1990, "A circular non-Newtonian Fluid Model: Part II - used in Micro-Elastohydrodynamic Lubrication," ASME JOT, 112, pp. 497-505.

19. Ling, F.B., Evans, H.P., and Snidle, R.W., 1989, "Micro-Elastohydrodynamic Lubrication of Circumferentially Finished Rollers: The Influence of Temperature and Roughness," ASME JOT, 111, pp. 730-736.

20. Lubrecht, A.A., 1987, "Numerical Solution of the EHL Line and Point Contact Problem Using Multigrid Techniques," PhD. Thesis, University of Twente, Enschede, The Netherlands. ISBN 90-9001583-3. 
21. Lubrecht, A.A., ten Napel, W.E., Bosma, R., 1988, "The Influence of Longitudinal and Transverse Roughness on the Elastohydrodynamic Lubrication of Circular Contacts", ASME JOT, 110, pp. 421-426.

22. Lubrecht, A.A., Venner, C.H., Lane, S., Jacobson, B., and Ioannides, E., 1990, "Surface Damage - Comparison of Theoretical and Experimental Endurance Lives of Rolling Bearings," Proceedings of the 1990 Japan International Tribology Conference, Nagoya, Japan, 1, pp. 185-190.

23. Lubrecht, A.A., and Venner, C.H., 1992, "Aspects of two-Sided Surface Waviness in an EHL Line Contact," Proceedings of the 1992 Leeds-Lyon Symposium on Tribology, Leeds, Elsevier, pp. 205-214.

24. Morales Espejel, G.E., 1993, "Elastohydrodynamic lubrication of smooth and rough surfaces," PhD. Thesis, University of Cambridge, Department of engineering.

25. Osborn, K.F., Sadeghi, F., 1992, "Time Dependent Line EHD Lubrication using the Multigrid/Multilevel Technique", ASME JOT, 114, pp. 68-74.

26. Roelands, C.J.A., 1966, "Correlational Aspects of the Viscosity-Temperature-Pressure Relationship of Lubricating Oils," PhD. Thesis, Technical University Delft, Delft, The Netherlands, (V.R.B., Groningen, The Netherlands).
27. Venner, C.H., ten Napel, W.E., and Bosma, R., 1990, "Advanced Multilevel Solution of the EHL Line Contact Problem," ASME JOT, 112, pp. 426-432.

28. Venner, C.H., Lubrecht, A.A., and ten Napel, W.E., 1991, "Numerical Simulation of the Overrolling of a Surface Feature in an EHL Line Contact," ASME JOT, 113, pp. 777-783.

29. Venner, C.H., 1991, "Multilevel Solution of the EHL Line and Point Contact Problems," $\mathrm{PhD}$. Thesis, University of Twente, Enschede, The Netherlands. ISBN 90-9003974-0.

30. Venner, C.H., and Lubrecht, A.A., 1994, "Transient Analysis of Surface Features in an EHL Line Contact in the case of Sliding," ASME JOT, 116, pp. 186-193.

31. Venner, C.H. and Lubrecht, A.A. "Numerical Simulation of a Transverse Ridge in a Circular EHL Contact, under rolling/sliding", to appear in ASME JOT.

32. Wedeven, L.D., and Cusano, C., 1979, "Elastohydrodynamic Film Thickness Measurements of Artificially Produced Surface Dents and Grooves," ASLE Trans., 22, pp. 369-381. 